\title{
LA DOCTRINA DEL FAIR USE FRENTE A LOS RETOS IMPUESTOS POR EL ENTORNO DIGITAL. ESTUDIO DEL CASO GOOGLE BOOKS
}

\author{
Luisa Fernanda Herrera Sierra*-**
}

How the law adapts to the continuous change in the opportunities and constraints which confront each of us in daily life, largely determines the shape and direction of social existence itself ${ }^{1}$.

El 16 de octubre de 2015, la Corte de Apelaciones de Estados Unidos decidió la apelación formulada por la Asociación de Autores Norteamericana contra la sentencia proferida el 14 de noviembre de 2013 por la Corte del Distrito de Nueva York en el caso The Authors Guild, Inc. and Betty Miles, Joseph Goulden, and Jim Bouton v. Google Inc. ${ }^{2}$. Este caso ofrece nuevas interpretaciones y perspectivas en

* Abogada de la Universidad Externado de Colombia. Llm en Propiedad Intelectual de la Universidad Queen Mary, University of London. Actualmente se desempeña como docente investigadora del Departamento de Propiedad Intelectual de la Universidad Externado de Colombia. Correo-e: luisa.herrera@uexternado.edu.co Fecha de recepción: 4 de septiembre de 2015. Fecha de aceptación: 15 de octubre de 2015. Para citar el artículo: Herrera S., L. F. La doctrina del fair use frente a los retos impuestos por el entorno digital. Estudio del caso Google Books. Revista La Propiedad Inmaterial n. ${ }^{\circ}$ 20, Universidad Externado de Colombia, julio-diciembre 2015, pp. 57-83. DOI: http://dx.doi.org/10.18601/16571959. $\mathrm{n} 20.04$

** La autora agradece al Dr. Ernesto Rengifo, Director del Departamento de la Propiedad Intelectual de la Universidad Externado de Colombia, por ser quien apoyó y motivó la elaboración del presente artículo.

1. Adelstein Richard and Peretz Steven, "The competition of technologies in markets for ideas: Copyright and fair use in evolutionary perspective", International Review of Law and Economics, 1985. Disponible en: http://works.bepress.com/cgi/viewcontent.cgi?article $=1015 \&$ context $=$ adelstein $\&$ sei-redir $=1$

2. The Economist comenta la decisión al manifestar que "no todo debe ser para enriquecer al autor" ("it's not all about enriching authors"); se trata, dice esta prestigiosa publicación, de garantizar el acceso al conocimiento. The Economist, "Google Books wins a court battle", Disponible en: http://www.economist.com/blogs/democracyinamerica/2015/10/ 
el análisis de la doctrina del fair use en el entorno digital. El propósito del presente artículo es analizar las decisiones de primera y segunda instancia, así como estudiar sus posibles implicaciones en el entorno digital ${ }^{3}$.

La evolución del derecho de autor demuestra la gran importancia que han adquirido los mecanismos de difusión, como factores determinantes del amoldamiento de las normas a las nuevas realidades y como herramientas que permiten el acceso al conocimiento. El proyecto de Google Books se ha constituido como un poderoso instrumento de difusión, cuyas implicaciones trascienden aquellos efectos generados por la imprenta, lo cual genera ciertas inquietudes que marcarán el transcurrir del análisis que se plantea en el presente documento. En efecto, es importante consultar los alcances de la doctrina del transformative use, cuya aceptación en la jurisprudencia ha implicado una ampliación de la doctrina del fair use, aspectos estudiados en el siguiente acápite. Además, aún son imprecisos los métodos o criterios a tener en cuenta en el estudio del uso transformativo. Y por último, conviene indagar si, dada la reciente jurisprudencia en la materia y el devenir histórico del derecho de autor, es posible afirmar que los mecanismos de difusión han adquirido mayor importancia que el mismo derecho de autor.

Con el objetivo de atender dichos interrogantes, el presente documento se estructura en seis acápites: en primer lugar, se expone la evolución jurisprudencial del fair use y el transformative use; luego, brevemente se desarrollan los fundamentos fácticos y jurídicos aducidos en la sentencia Google Books de 2013, así como en la de segunda instancia de octubre de 2015, para enseguida examinar a fondo la doctrina del transformative use, con base en la cual las Cortes construyeron su argumentación; posteriormente se evalúan los posibles efectos de las decisiones en el sistema bibliotecario; y finalmente se plantean algunas conclusiones derivadas del análisis.

\section{DE LOS ORÍGENES DEL DERECHO DE AUTOR A LA DOCTRINA DEL FAIR USE}

Resulta fundamental tener presente la evolución de la difusión del conocimiento como gran motor del progreso de la humanidad, las artes y la ciencia. Inicialmente, solo unos cuantos podían acceder al conocimiento y comprenderlo; luego, con el advenimiento de nuevos mecanismos de difusión, tales como la imprenta,

words-masses?utm_content=bufferae $16 c \& u t m \_m e d i u m=$ social $\& u t m \_s o u r c e=f a c e b o o k$. com\&utm_campaign=buffer

3. Luisa Fernanda Herrera Sierra, "Estudio del Caso Google Books y efectos del fair use en el entorno digital", disponible en: http://propintel.uexternado.edu.co/estudiodel-caso-google-books-y-efectos-del-fair-use-en-el-entorno-digital/ 
aquellos receptores de información documentada se fueron incrementando. Así mismo, a raíz del Estatuto de la Reina Ana de 1710, en el derecho anglosajón, el control de las obras dejó de estar monopolizado por los monarcas, quienes otorgaban "privilegios" a ciertas imprentas para reproducir las obras. Mediante el mencionado estatuto se otorgaron derechos exclusivos en cabeza de autores y editores para la reproducción de libros ${ }^{4}$. Estos derechos se consagraron bajo una limitación temporal, garantizando así lo que la doctrina ha denominado como el quid pro quo social en el derecho de autor. Es decir, se empieza a concebir este derecho como un conjunto de prerrogativas otorgadas temporalmente con el objetivo de promover la creación y además de incentivar la difusión y el acceso al conocimiento.

Así las cosas, el derecho de autor evoluciona para convertirse en un derecho de propiedad inmaterial, que al igual que el que recae sobre bienes físicos, no es absoluto y se encuentra limitado por ciertos intereses y criterios de política pública. De ahí la importancia de las excepciones y limitaciones al derecho de autor, las cuales garantizan un balance constante entre derechos. Dichas excepciones han sido concebidas a través de dos modelos jurídicos diferentes: aquellos que contemplan una enumeración taxativa de las excepciones o circunstancias que admiten el uso de las obras sin autorización del autor, como sucede en los países de la Unión Europea; y otros sistemas que, como el norteamericano, han adoptado la doctrina del fair use, de acuerdo con la cual cada caso debe analizarse en concreto para determinar si el uso dado a la obra es considerado como una infracción al derecho de autor. Este último sistema es al que se contrae el presente análisis, por cuanto fue el aplicado por la Corte en el fallo.

El Congreso de Estados Unidos, convencido de la importancia de admitir el uso de las obras en ciertas circunstancias con el fin de promover la creatividad y la originalidad ${ }^{6}$, adopta la doctrina del fair use en la Sección 7 del Copyright Act de 1976, disposición que consagró cuatro factores que deben ser analizados para determinar si el uso de la obra ha sido justo:

1. El propósito y carácter del uso, análisis que debe considerar si tal uso es de naturaleza comercial o con propósitos educativos sin ánimo de lucro;

2. La naturaleza de la obra protegida;

3. La proporción utilizada, en términos de cantidad y calidad, y en relación con la totalidad de la obra

4. Ibíd.

5. R. Deazley, Rethinking Copyright: History, Theory, Language, p. 13.

6. Robinson v. Random House, Inc, 1995, citado en Ashten Kimbrough, "Transformative use vs. market impact: Why the fourth fair use factor should not be supplanted by transformative use as the most important element in a fair use analysis", Alabama Law Review, 2012, p. 625. 
4. El efecto de su uso en mercados potenciales o en el valor de la obra protegida ${ }^{7}$.

Si bien esta doctrina ha sido criticada por la amplitud de la flexibilidad que imprime, en cuanto se le endilga una seria limitante del derecho de autor, en el análisis de las posibles situaciones de infracción, recientemente también se ha reconocido su utilidad al enfrentar los nuevos retos impuestos por la evolución tecnológica en relación con la necesidad del acceso a la cultura.

Hay quienes esgrimen que Europa debería acoger la doctrina del fair use en la medida en que le otorgaría mayor autonomía interpretativa al juez. Al respecto Jonathan Griffiths sostiene: "El desarrollo de dicha teoría no solo permitiría aliviar la inflexibilidad de aquellas predominantes corrientes europeas, sino que además, reduciría la ventaja competitiva que tiene Estados Unidos sobre Europa, y además aseguraría un grado de armonización dado el aumento de jurisdicciones que han acogido la doctrina en análisis".

Ahora bien, las decisiones que se comentan trascienden el estudio teórico de la doctrina del fair use. En efecto, las sentencias no solo consagran los rasgos característicos del nuevo derecho de autor, interpretado en un entorno digital, sino que además identifican aquellos nuevos visos que el concepto de transformative use imprime en la interpretación jurídica de los usos justos. Aunque este concepto será estudiado más adelante, es de resaltar desde ya la gran importancia que ha adquirido en la evaluación de los factores integrantes de la doctrina del fair use.

Efectivamente, a partir del caso Campbell v. AcuffRose Music, Inc., las Cortes incluyeron dicha doctrina como factor predominante en el análisis del fair use. En esta sentencia, la Suprema Corte concluyó que la adaptación a versión rap de la reconocida canción Pretty Woman del artista Roy Orbison realizada por el grupo Live Crew, consistía en una parodia de la versión original y en consecuencia debía ser considerado como un uso transformativo. Así pues, dicha decisión agregó nuevos elementos en el análisis de los usos justos, elementos que serán comentados más adelante dado que sirvieron de fundamento a la Corte al determinar si la difusión de las obras a través de Google Books consistía en un uso transformativo.

Así mismo, la doctrina ha definido el uso transformativo como aquella modificación sustancial y formal de una obra, convirtiéndola en una nueva creación:

7. Traducción libre.

8. Jonathan Griffiths, "Unsticking the centre-piece - The liberation of European copyright law?”, Disponible en: http://www.jipitec.eu/issues/jipitec-1-2-2010/2617/JIPITEC\%20 2\%20- Traducción libre del siguiente extracto: "The development of such a doctrine would not only help to alleviate the inflexibility currently prevailing in the European copyright system, but may also go some way to reducing the competitive advantage that the fair use doctrine may grant the US over Europe and would secure a degree of harmonisation with the increasing number of jurisdictions adopting fair use-type provisions around the world". 
"If $[. .$.$] the secondary use adds value to the original - if the quoted matter is used as$ raw material, transformed in the creation of new information, new aesthetics, new insights and understandings - this is the very type of activity that the fair use doctrine intends to protect for the enrichment of society"'.

Con el advenimiento de los nuevos medios tecnológicos e informáticos de difusión, a través de los cuales se accede a las obras literarias, el contexto inicial en el cual nació el derecho de autor se ha ido modificando, imponiendo nuevos retos; así, "cada consumidor que se conecta es un autor, un editor y un infractor en potencia, las tres cosas al mismo tiempo o en diferentes momentos" ${ }^{10}$. Por ello el derecho de autor se ha ido moldeando a las nuevas realidades, de ahí la importancia de valorar con cuidado los criterios que deben utilizar tanto el juez como el legislador cuando consideren que un derecho se ha infringido o cuando el uso de una obra en el entorno digital cae en la órbita de las limitaciones o excepciones.

En este contexto, ciertas tendencias defienden la apropiación e imitación de ideas con el fin de garantizar la difusión y el acceso al conocimiento. Algunos aseguran que esto generaría mayor creación por cuanto quien se apropia de una idea ajena adquiere la responsabilidad de mejorarla, desarrollarla y convertirla en una nueva creación. Seth Godin, por ejemplo, sostuvo en el blog "Questions Worth Asking" de la página web TED: "With the ability to steal comes responsibility. Not just the responsibility to synthesize something better than what you started with, but the obligation to relentlessly seek out the next thing worth stealing. We've created a bucket line. Our economy is a long line of people handing ideas up and down the line, improving and customizing at each step. When you stop seeking and merely consume, you let us all down"11.

Podría considerarse que la aplicación de la doctrina del transformative use refleja en cierta medida aquella tendencia, por cuanto admite el "uso creativo" 12 de obras protegidas siempre y cuando el propósito de la obra original sea transformado, permitiendo a la vez que se cumplan los objetivos primordiales del derecho de autor: el acceso al conocimiento y la promoción de las artes, la ciencia y la cultura. Esto se complementa además con los desarrollos de la doctrina del fair use, la cual ha sido considerada como aquel motor generador de mayor creación

9. Pierre N. Leval, Towards a fair use standard, citado en Ashten Kimbrough, "Transformative use vs. market impact: Why the fourth fair use factor should not be supplanted by transformative use as the most important element in a fair use analysis", Alabama Law Review, 2012, p. 625.

10. Ralph Oman, citado en "Foro de expertos sobre el derecho de autor, Hacia un nuevo contrato. El derecho de autor en el entorno digital”, p. 17.

11. Artículo disponible en: http://blog.ted.com/2014/02/03/the-big-mistake-we-allmake-about-ideas/

12. Ashten Kimbrough, "Transformative use vs. market impact: Why the fourth fair use factor should not be supplanted by transformative use as the most important element in a fair use analysis", Alabama Law Review, 2012, p. 626. 
e innovación, a partir de obras ya existentes. Sobre el particular, Mark A. Fisher manifestó: "the concept behind fair use is that creativity often requires the use of others' works for the expression of ideas" ${ }^{13}$. No obstante lo anterior, debe reconocerse también la importancia de la escasez como factor originario de la creación, dado que esta última nace ante la necesidad de innovar. En otras palabras, ante la exclusión se incentiva tanto a los antiguos autores como a los nuevos, a innovar y a desarrollar nuevas ideas. En cambio, el uso irrestricto de obras podría generar no solo efectos nefastos en el proceso creativo de un país, sino además la violación flagrante y constante de derechos.

Analizado lo anterior, será necesario examinar los nuevos alcances que las decisiones de Google Books le otorgan al transformative use en el entorno digital. Para ello, a continuación se estudian en detalle las sentencias.

\section{Análisis de la Sentencia del i4 De noviembre de 20i3, Corte del Distrito de Nueva York}

\section{Hechos}

1. En el año 2004, mediante la suscripción de acuerdos con varias bibliotecas, Google dio inicio a su proyecto de crear una biblioteca digital en internet.

2. En virtud de dichos acuerdos, las bibliotecas partícipes del proyecto permitieron que Google escaneara más de 20 millones de obras pertenecientes a sus colecciones, con el fin de difundir dichas copias digitales en internet.

3. Google realizó el cambio de formato de las obras y su difusión en internet sin previa autorización por parte de los titulares de derechos de autor.

4. Entre las obras escaneadas, algunas estaban protegidas por derechos de autor y otras se encontraban dentro del dominio público.

5. Aproximadamente el $93 \%$ de los libros no eran de ficción y la gran mayoría se encontraban fuera del comercio.

6. Google publicó las obras bajo un sistema denominado "snippets", mediante el cual se hacían visibles extractos, por grupos de páginas. Con ello Google evitaba que los usuarios consultaran la totalidad del libro. Así mismo, los usuarios no podían modificar el número ni el orden de extractos disponibles al público. A manera de ejemplo, si un usuario ingresaba la totalidad del texto de un capítulo, o gran parte de este en el buscador, no podía acceder al capítulo y a lo sumo obtenía como resultado de su búsqueda extractos relevantes con frases o palabras objeto de consulta.

13. Mark A. Fischer, Duane Morris llp, “All's fair in copying the world's books", disponible en: http://www.mediabizbloggers.com/mark-fischer/Alls-Fair-in-Copying-theWorlds-Books----Mark-Fischer.html 
6. Google había establecido un mecanismo mediante el cual los usuarios del buscador podían ubicar los libros escaneados a partir de palabras o frases vinculadas con el texto escaneado. Una vez el usuario había ingresado al texto del libro, tenía la posibilidad de ingresar a la información del mismo y el sistema proveía además la lista de tiendas o librerías donde el usuario podía encontrar y adquirir el libro.

7. Mediante este sistema, se permitió a aquellas bibliotecas partícipes del proyecto de Google Books acceder y descargar la totalidad de los textos que se encontraban dentro de sus colecciones, no siendo posible descargar contenidos pertenecientes a las colecciones de otras bibliotecas.

8. En el año 2005, cinco editores miembros de la Asociación de Editores Americanos (Association of American Publishers - AAP) y el gremio de autores americano (Authors Guild) instauraron una acción de clase, alegando infracción de derechos de autor a raíz de la divulgación de copias digitales de las obras sin previa autorización de los titulares de derechos.

9. En noviembre de 2009 las partes suscribieron un acuerdo de conciliación.

10. En el mencionado acuerdo, Google adquirió las siguientes obligaciones: 1) al pago de la suma de 125 millones de dólares a los titulares de los derechos sobre las obras escaneadas; 2) al pago de las costas del proceso a los demandantes, y 3) a la creación de un registro de derechos de autor sobre los libros escaneados.

11. Lo anterior se condicionó al cumplimiento de ciertas prerrogativas a favor de Google: 1) la posibilidad de difundir las copias digitales de los libros en internet y bajo el sistema "snippets"; 2) permitir el acceso al 20\% de las obras por parte de los usuarios del buscador; 3 ) la posibilidad de ofrecer a la venta dichas versiones digitales.

12. En el acuerdo de conciliación se estipuló que los titulares de derechos de autor recibirían el $63 \%$ de las ganancias generadas a raíz de la comercialización de las copias digitales de sus obras.

13. En marzo de 2011, el juez Denny Chin rechazó el texto del acuerdo presentado por las partes, decisión que fundamentó de la siguiente forma:

While the digitization of books and the creation of a universal digital library would benefit many, the ASA would simply go too far. It would permit this class action - which was brought against defendant Google Inc. ("Google") to challenge its scanning of books and display of "snippets" for on-line searching - to implement a forward-looking business arrangement that would grant Google significant rights to exploit entire books, without permission of the copyright owners. Indeed, the ASA would give Google a significant advantage over competitors, rewarding it for engaging in wholesale copying of copyrighted works without permission, while releasing claims well beyond those presented in the case. 
Accordingly, and for the reasons more fully discussed below, the motion for final approval of the ASA is denied ${ }^{14}$.

14. En octubre de 2012, la asociación de editores americana suscribió acuerdo de conciliación con Google. En virtud de dicho acuerdo, la asociación acordó trabajar en conjunto con Google con el fin de difundir obras digitales en internet a través de Google Books y Google Play. Este acuerdo privado no afectó el litigio entre Google y el gremio de autores.

15. En mayo de 2013, la Corte de Apelaciones del Segundo Circuito ${ }^{15}$ reconoció la legitimación por activa de "The Authors Guild” y remitió el asunto a la Corte del Distrito de Nueva York para su conocimiento.

\section{Consideraciones de la Corte}

\section{a. Las ventajas de Google Books expuestas por la Corte}

Previo al análisis del fair use, la Corte trae a colación los beneficios del proyecto de Google Books. En primer lugar, la introducción de un nuevo sistema de búsqueda de libros estimula tanto la investigación individual como aquella derivada de la dinámica entre bibliotecas. Así pues, se promueve los métodos del "data mining" o "text mining", mediante los cuales, expertos podrían identificar y analizar las tendencias gramaticales y semánticas. De esta forma, podrían establecerse interesantes indicios acerca de la acogida cultural y social de ciertas expresiones o palabras, acerca de su historia y la frecuencia de su uso en la literatura. En cuanto a las bibliotecas, el proyecto de Google Books les otorga la posibilidad técnica de ofrecerle al usuario copias digitales de los libros que se encuentran en sus repositorios, así como sistemas digitales de búsqueda.

Además de la difusión del conocimiento y el acceso a la literatura, Google permite a discapacitados visuales acceder a formatos acordes con sus necesidades, garantizando así un acceso igualitario y equitativo al conocimiento.

Google permite la conservación de la literatura, permitiendo que mediante otros formatos esta sea difundida. Esto evita las desventajas del formato físico de los libros y su carácter perecedero, tales como el estancamiento de las obras en bibliotecas, sin alguna posibilidad de ser aprovechadas y difundidas.

Finalmente, Google parece crear mayores fuentes de ingreso para las compañías editoriales, comoquiera que su sistema permite a usuarios identificar los

14. Juez Denny Chin, United States District Court Southern District of New York, The Authors Guild et al. v Google Inc, decisión del 22 de marzo de 2011, disponible en: http://www.nysd.uscourts.gov/cases/show.php?db=special\&id=115

15. Ibíd. 
vendedores de los libros objeto de búsqueda. Así, si el usuario se ha interesado a partir de la muestra electrónica disponible, este estaría en la capacidad de obtener la información acerca de dónde y cómo adquirir la totalidad del texto.

\section{b. La doctrina del fair use a la luz de la interpretación de la Corte}

El razonamiento de la Corte se deriva en esencia de los fines del derecho de autor, los cuales han sido reconocidos uniformemente por la jurisprudencia americana en casos como Campbell v. Acuff-Rose Music, Inc. y Cariou v. Prince. La Corte ha manifestado que los derechos de autor no solo promueven la creación al otorgar derechos exclusivos sobre la explotación de las obras, sino que además permiten la difusión y el progreso de las artes, la ciencia y la cultura. Así mismo, la Suprema Corte de Estados Unidos ha sostenido que a través de la doctrina del fair use o uso justo es posible garantizar el cumplimiento de dichos objetivos puesto que permite el uso de las obras protegidas en circunstancias especiales que justifiquen dicho uso.

De lo anterior puede concluirse la naturaleza abstracta de la doctrina. En efecto, la jurisprudencia ha reconocido que se trata de una doctrina cuyos factores no son taxativos ni exclusivos, y por tanto requiere de un análisis on a case by case basis. Ahora bien, al tratarse de una defensa que puede ser alegada por el demandado, este tiene la carga probatoria de demostrar el uso justo de la obra.

Habiendo señalado lo anterior, la Corte procedió a analizar cada uno de los criterios.

\section{i. La naturaleza del uso de las obras por parte de Google Books}

En los desarrollos jurisprudenciales de esta doctrina, las Cortes han diferenciado los usos que otorgan adicionales características a la obra, modificando el mensaje y creando nuevos significados, de aquellos usos que solo suplantan o reemplazan la obra original. Así, las Cortes se han referido a aquellos casos en los que las obras son "transformative" y aquellos en los que las obras "supersedes or supplants the original creation".

La Corte concluye que el uso que Google hace de los libros es principalmente transformative comoquiera que con la digitalización de los libros y la toma de su contenido en un exhaustivo índice de palabras se permite a lectores, académicos, investigadores y otros ubicar los libros. Pero con ello no se busca suplantar las obras originales. La Corte llega a la anterior conclusión apoyándose en los casos Perfect 10, Inc. v. Amazon.com y Kelly v. Arriba Soft Corp.

Así, de acuerdo con sus consideraciones, la Corte sostiene que el uso no pretende suplantar o sustituir la obra original ya que el sistema de Google se encuentra diseñado para la búsqueda de los libros y no para su lectura; esto le otorga mayor 
valor al trabajo protegido, permitiendo no solo un mayor reconocimiento por parte de los lectores, sino además nuevos usos de la obra original. Vale la pena hacer referencia textual a lo sostenido por el Juez Denny Chin: "Google Books digitizes books and transforms expressive text into a comprehensive word index that helps readers, scholars, researchers, and others find books. [...] Google Books does not supersede or supplant books because it is not a tool to be used to read books".

Señala la Corte que si bien existen propósitos económicos y de lucro detrás del proyecto de Google Books, también es cierto que no se trata de un sistema construido para vender libros y que por el contrario, consiste en un esquema que incentiva la investigación y la difusión de la cultura.

ii. Naturaleza de las obras

En esencia, respecto de este punto la Corte subsume el análisis al determinar que se trata de un uso transformativo, por lo cual, independiente de la naturaleza de las obras utilizadas en el sistema de búsqueda, se ha configurado un uso justo de las mismas.

iii. Proporción o porcentaje de los extractos utilizados

En casos como Sony Corp. of Am. v. Universal City Studios y Bill Graham Archives, las Cortes han determinado que en ciertas ocasiones la copia completa de una obra puede considerarse como un uso justo. Además, aunque Google escanea la totalidad del libro, solo admite el acceso a ciertos extractos de las obras como resultado de las búsquedas.

iv. El efecto del uso en el mercado sobre el valor de la obra protegida

La Corte sostiene que pese a que los demandantes argumentan que las obras ofrecidas por el sistema de búsqueda de Google Books reemplaza el mercado de libros físicos, esto no coincide con el verdadero propósito para el cual se encuentra diseñado el sistema. En efecto, Google no ofrece a la venta los libros; los resultados de búsqueda solo permiten a los usuarios acceder a extractos, no a la totalidad de las obras, y además otorga la posibilidad de ubicar las editoriales o compañías vendedoras de los libros en cuestión.

\section{Decisión de la Corte}

Finalmente, la Corte concluye que el uso de las obras por parte de Google Books coincide con la doctrina del fair use, no solo porque se encuentran cumplidos los factores mencionados anteriormente, sino también porque el proyecto de- 
sarrollado permite un mayor progreso de las ciencias y las artes. Así mismo, en virtud de decisiones como Authors Guild, Inc. v. HathiTrust, las librerías que le concedieron a Google el acceso a los libros y luego hicieron uso de las copias digitales no infringen derechos de autor. En efecto, en este caso se trata de un uso justo, destinado a la educación y a la difusión de la cultura. Al respecto, la Corte manifiesta:

Google provides the libraries with the technological means to make digital copies of books that they already own. The purpose of the library copies is to advance the libraries' lawful uses of the digitized books consistent with the copyright law. The libraries then use these digital copies in transformative ways. They create their own full-text searchable indices of books, maintain copies for purposes of preservation, and make copies available to printdisabled individuals, expanding access for them in unprecedented ways. Google's actions in providing the libraries with the ability to engage in activities that advance the arts and sciences constitute fair use.

En el caso HathiTrust, la Corte determinó que el uso de obras originales por parte de Google, en virtud de acuerdos celebrados con ciertas universidades con el objetivo de desarrollar un repositorio de copias digitales de libros en internet, constituía un uso transformador. Se consideró, entonces, que la difusión de dichas copias cumplía con fines educativos completamente diferentes al propósito expresivo y creativo de las obras originales comoquiera que con ello se pretendía ofrecer versiones de audio o táctiles a los discapacitados visuales. La esencia de la doctrina del transformative use fue plasmada por la Corte en Hathi Trust al describir el propósito del proyecto desarrollado por Google: "provides social benefit by shedding light on an earlier work, and in the process, creates a new one".

\section{Análisis de la Decisión de la Corte de Apelaciones DEL I6 DE OCTUBRE DE 2OI5 $5^{[16]}$}

En sus consideraciones, el Juez Pierre Leval confirma las bases fundamentales del fallo de primera instancia. En efecto, la Corte de Apelaciones reconoce que se trata de un uso transformativo de las obras puesto que Google convierte las obras en una herramienta de búsqueda. Ahora, este nuevo pronunciamiento resulta fundamental dado que reconoce de manera clara, directa y expresa que el proyecto de Google Books puede generar perjuicios económicos al autor, pero justifica dichos perjuicios en los beneficios sociales derivados del acceso a diversas obras de literatura.

16. Authors Guild v. Google, Inc. United States Court of Appeals for The Second Circuit - October 16, 2015. 
$\mathrm{Al}$ iniciar el análisis, la Corte hace un especial reconocimiento a la importancia del caso: "Este conflicto de derechos de autor somete a prueba los límites del uso justo" ${ }^{17}$. En efecto, el caso pone bajo prueba los límites del derecho de autor, y por ende, todas aquellas excepciones y limitaciones establecidas por los ordenamientos jurídicos, tanto en el sistema anglosajón como en el continental.

La Corte, entonces, se ve abocada a poner en una balanza todos los intereses involucrados, pero para ello hace una advertencia inicial: "dar a los autores un control absoluto sobre sus obras crearía, en algunas circunstancias, no expansiones del conocimiento sino limitaciones al mismo" ${ }^{18}$. Lo anterior, lo fundamenta al argumentar que el libre acceso incentiva mayor creación.

Sin embargo, a diferencia de la primera instancia, esta Corte aclara que no todo cambio en la obra anterior debe ser considerado como un uso transformativo, son solo aquellas transformaciones que modifican el propósito original de la obra. Si bien Google Books copia la totalidad de la obra, la Corte aclara que lo relevante es la cantidad puesta a disposición del público. De ahí la gran importancia del concepto de "snippets" o extractos. Los usuarios no pueden acceder a la totalidad de la obra sino a extractos.

Tal como se mencionó atrás, la Corte reconoce que el acceso a extractos también puede ocasionar perjuicios económicos y pérdidas en las ventas del autor. No obstante esta consideración, el beneficio social que se obtiene es mucho mayor. Adicionalmente, la Corte agrega un elemento al cuarto factor que no se encuentra expresamente consagrado en la norma. Exige que el daño económico sea relevante y evidente. Lo anterior es manifestado en los siguientes términos:

But the possibility, or even the probability or certainty, of some loss of sales does not suffice to make the copy an effectively competing substitute that would tilt the weighty fourth factor in favor of the rights holder in the original. There must be a meaningful or significant effect "upon the potential market for or value of the copyrighted work.

[...] that snippet view could provide a significant substitute for the purchase of the author's book. [...] There is no basis in the record to impose liability on Google for having lawfully made a digital copy for a participating library so as to enable that library to make non-infringing use of its copy, merely because of the speculative possibility that the library may fail to guard sufficiently against the dangers of hacking, as it is contractually obligated to do. [...] Nor, on this record, is Google a contributory infringer.

17. Traducción libre del siguiente texto: "This copyright dispute tests the boundaries of fair use".

18. Traducción libre del siguiente texto: "giving authors absolute control over all copying from their works would tend in some circumstances to limit, rather than expand, public knowledge". 
Y concluye la Corte reiterando que los extractos a los cuales acceden los usuarios no ofrecen realmente una apreciación o una experiencia significativa del libro en su conjunto:

While it is true that Google's snippets display a fragment of expressive content, the fragments it displays result from the appearance of the term selected by the searcher in an otherwise arbitrarily selected snippet of text. Unlike the reading experience that the Google Partners program or the Amazon Search Inside the Book program provides, the snippet function does not provide searchers with any meaningful experience of the expressive content of the book.

Del anterior resumen de la decisión de la Corte de Apelaciones se concluye que el transformative use sigue siendo un factor determinante en las decisiones y ahora cuenta con el aval de instancias superiores. No obstante la Corte hace un loable esfuerzo por aclarar que no todo cambio en la obra original implica un uso justo de la misma, dejando sin embargo cierta ambigüedad en el análisis, por cuanto reconoce que en el caso en concreto no hubo cambios significativos, comoquiera que se modificó únicamente el formato, y se ofrece un acceso limitado a las obras. Pero está claro que la obra sigue siendo la misma, lo modificado fue su propósito y la cantidad disponible al público. Adicionalmente, requiere que exista un perjuicio económico significativo, ingrediente que no se encuentra en la norma y que ahora puede hacer eco en futuras decisiones jurisprudenciales. Aunque la Corte manifiesta adoptar una posición que le da prioridad a los beneficios sociales, al conocimiento y al estímulo de la creación, sigue reinando la ambigüedad y la incertidumbre a la hora de establecer cuándo un uso será realmente transformador y cuando este no implicará un "significativo" detrimento en las ventas del autor o titular de derechos.

\section{Análisis del caso y las posiciones asumidas por las Cortes. Comentarios acerca de la doctrina DEL TRANSFORMATIVE USE}

En esencia, la doctrina del fair use, tal como ha sido desarrollada por las Cortes en casos como el de Arica Inst, Inc. v. Palmer, permite identificar, de conformidad con los hechos del caso en concreto, si los fines primordiales del derecho de autor se cumplen de mejor forma al admitir ciertos usos de las obras. El análisis realizado por las Cortes al aplicar dicha doctrina se encuentra condensado en la primera frase de la Sección 107 del Copyright Act de 1976, mencionada anteriormente: "the fair use of a copyrighted work [...] for purposes such as criticism, comment, new reporting, teaching [...] scholarship, or research, is not an infringement of copyright". Las Cortes deben estudiar entonces, no solo si se trata de un caso de fair use, sino 
también si el uso se encuentra destinado a cumplir un propósito completamente diferente para el cual fue creada la obra original ${ }^{19}$.

En cuanto al examen del propósito, resulta interesante consultar la evolución de la jurisprudencia americana en el análisis de esta doctrina. El primer caso donde la Suprema Corte de Estados Unidos hubo de referirse al fair use fue Sony Corp of America v. Universal City Studios, Inc, decisión en la cual la Corte expresó que para admitir un determinado uso, este debe cumplir las características del denominado "productive use". En este caso, el Juez Blackmun sostuvo: "although such transformative use is not absolutely necessary for a finding of fair use [...] the goal of copyright, to promote science and the arts, is generally furthered by the creation of transformative Works". Es decir, si bien un uso transformador de la obra no resulta ser un requisito sine qua non para concluir que se trata de un uso justo, sí es cierto que la creación de obras transformadoras permite incentivar los principales objetivos del derecho de autor: la ciencia y las artes. Así mismo, se determinó que en casos donde se evidencie mayor transformación en la obra, la necesidad de consultar otros criterios, tales como el propósito comercial del uso, será mucho menor.

La Corte reconoció en esta decisión que si bien no todos los usos productivos (o productive uses) podrían ser justos, únicamente esta categoría de usos, es decir, aquellos que agregan adicionales características a la obra original y que benefician considerablemente al público, podrían ser considerados como justos. Posteriormente, en el caso Campbell v. Acuff-Rose Music, Inc, la Corte comienza a considerar con mayor profundidad el examen del propósito, y lo que venía desarrollando como el "productive use", lo denomina "transformative use". Este último concepto es explicado por la Corte en Campbell al definir aquello que no puede ser considerado como transformativo, es decir, meras reproducciones de la obra original: "a work composed primarily of an original, particularly its heart, with little added or changed, is more likely to be a merely superseding use, fulfilling demand for the original'20.

Cabe reiterar el grado de importancia que ha adquirido en las Cortes el análisis del transformative use. En efecto, se ha sostenido que cada uno de los factores integrantes de la doctrina del fair use debe interpretarse de acuerdo con el grado transformativo del uso de la obra. Así, cuando las Cortes se han ocupado de determinar el porcentaje del uso de las obras (correspondiente al tercer factor ya comentado atrás), han determinado que un uso transformativo permitiría la copia de grandes porcentajes de la obra ${ }^{21}$. Esta evolución de la doctrina eviden-

19. Halpern Sheldon, Seymore Sean and Port Kenneth, "Fundamentals of United States Intellectual Property Law”, 4. ${ }^{a}$ ed., Wolters Kluwer, uK, 2012, p. 90.

20. Ibíd.

21. Ibíd. 
ció un gran cambio de posiciones desde la sentencia de Sony Corp of America v. Universal City Studios, Inc, en la cual los factores edificadores del uso justo fueron analizados independientemente del uso transformativo. En efecto, aun cuando se tratara de un nuevo uso con adicionales características transformadoras de la obra, la Corte concentraba su argumentación en determinar si se cumplía cada uno de los factores del fair use ya mencionados.

Así mismo, resulta fundamental consultar los desarrollos jurisprudenciales del uso transformativo en las tecnologías de la información y buscadores de internet. En Perfect 10, Inc. v. Amazon.com and Google, la Corte Federal del Noveno Circuito consideró que el uso de imágenes del sitio web Perfect 10 era un uso justo ya que cumplían un propósito diferente, el cual era dirigir a los usuarios a otras páginas web a través de imágenes en miniatura que una vez seleccionadas se convertían en hipervínculos. Así entonces, a juicio de la Corte, este uso de las fotografías aseguraba mayor difusión y acceso por parte de los usuarios. En esencia, el pronunciamiento se centró en analizar los beneficios generados a la sociedad a partir del uso transformativo de la obra, para así considerar que se trataba de un uso justo.

Además, en AV ex rel. Vanderhye v. iParadigms, LLC, la Corte Federal del Cuarto Circuito consideró que el uso que iParadigms hacía de las obras de dos estudiantes era transformativo. Así, el uso de obras en su versión digital con el fin de someterlas en un software mediante el cual se suben a internet para detectar signos de plagio, no podría considerarse como violación a derechos de autor por cuanto el cambio de formato obedecía a una necesidad académica.

Ahora bien, es labor de los críticos estudiar esta evolución y consultar cuáles son las limitaciones y las ventajas o desventajas del devenir de la doctrina del uso justo. Sobre el punto, académicos como Michael Murray han sostenido que un determinado uso será transformativo cuando ha habido una modificación sustancial del propósito para el cual fue creada la obra original, y cuyos efectos sean benéficos para el público en general, garantizando así el cumplimiento de los fines primordiales de la doctrina del uso justo. Aunado a ello, se ha afirmado que no es necesario que el contenido o la forma de expresión sean modificados, siempre y cuando las funciones o finalidades de la obra original cambien completamente ${ }^{22}$.

No obstante, aún no se ha establecido con precisión cuáles son los criterios aplicables para determinar si un uso es transformativo, y su estudio depende de cada caso en concreto. Por ello, un sector de la doctrina sostiene que el uso transformativo no debería convertirse en el factor más importante del fair use. Ashten Kimbrough afirma que la aplicación de la doctrina resulta ser confusa 
por cuanto aún es imprecisa la definición de transformative y no es claro qué tan transformativo debe ser el uso de una obra para que este pueda ser catalogado como justo $^{23}$. Para justificar lo anterior, Kimbrough trae a colación dos casos que demuestran las inconsistencias derivadas de la doctrina, por cuanto ante hechos similares las Cortes encuentran interpretaciones jurídicas diferentes. Por un lado, en Nunez v. Caribbean International News Corp., la Corte del Circuito sostuvo que la adición de comentarios a fotos protegidas por el derecho de autor era considerado como un uso transformativo de las obras, y por tanto, justo. Por otro lado, en Psihoyosv. National Examiner, la misma Corte consideró que el uso de la foto de un carro por una revista no era transformador ya que no modificaba el propósito de la obra original.

Pese a la fuerte influencia de la doctrina del transformative use en la jurisprudencia, resulta interesante mencionar posiciones jurisprudenciales que evidencian ciertas limitaciones en la aplicación de la doctrina. Así por ejemplo, en Warner Bros. Entm't Inc. v. RDR Books, la Corte del Distrito de Nueva York concluyó que aunque The Harry Potter Lexicon constituía un uso transformador de la obra original, no podía ser considerado como un uso justo. La Corte explicó que la edición de una enciclopedia con la explicación de términos contenidos en la obra de Harry Potter debía catalogarse como un uso transformador debido a que su propósito no era de entretenimiento sino de mera referencia a la obra original. Ahora bien, dicho uso podría afectar considerablemente el mercado de obras derivadas de Rowling, desmotivando a los consumidores a adquirir dichas nuevas obras que el autor está en el derecho de desarrollar a partir del libro de Harry Potter.

La Corte en el caso de Warner Bross plasma en sus considerandos dos postulados fundamentales: en primer lugar, se reitera que bajo ninguna circunstancia es admisible la copia flagrante de obras originales; y en segundo término, se exalta la importancia de encontrar un adecuado balance entre los derechos de autor y la libertad de expresión de aquellos autores que crean a partir de obras ya existentes. Así pues, se consideró lo siguiente:

In striking the balance between the property rights of original authors and the freedom of expression of secondary authors, reference guides to works of literature should generally be encouraged by copyright law as they provide a benefit to readers and students; but to borrow from Rowling's overstated views, they should not be permitted to "plunder" the works of original authors [...] "without paying the customary price" [...] lest original authors lose incentive to create new works that will also benefit the public interest $t^{24}$.

23. Ashten Kimbrough, "Transformative use vs. market impact: Why the fourth fair use factor should not be supplanted by transformative use as the most important element in a fair use analysis", Alabama Law Review, 2012, p. 625.

24. Ibíd. 
Igualmente, en la sentencia de Cariou v Richard Prince, la Corte de Apelaciones aclaró que no todo uso modificador de la obra original puede ser catalogado como transformador. Esto sucede, por ejemplo, con aquellos libros que recogen series de televisión donde, pese a que existe un cambio de formato, no existen aditamentos sustanciales y en esencia el contenido conserva su naturaleza original ${ }^{25}$. Empero, la Corte concluyó finalmente que la adición de nuevos elementos y diseños que Prince introdujo en algunas de las fotos de Cariou era suficiente para concluir que se trataba de un uso transformador y, por tanto, justo. Aunque este último fue un caso diferente al de Google Books, sobre el reconocido arte de "appropriation" y cuyos principales representantes han sido artistas como Andy Warhol y Roy Linchestein, quienes han capturado en su arte imágenes de la vida real, dicha decisión constituye otra evidencia de la expansión que ha adquirido la interpretación de los usos justos.

En efecto, la Corte no solo expande dicha interpretación al darle una función protagónica en la decisión a lo que consideró como un uso transformativo, sino que además introduce el concepto de "reasonable observer" como elemento determinante del fair use. A juicio de la Corte, un uso será transformador cuando pueda considerarse que un observador razonable lo catalogaría como tal ${ }^{26}$. A todas luces este nuevo concepto extiende de manera imprecisa el margen de aplicación de la teoría, no siendo aún claros los efectos que esto podría generar en el futuro derecho de autor.

Ante esta realidad de la jurisprudencia, cabe indagar acerca de las fundamentaciones aducidas por las Cortes en el caso Google y que en este escrito se analiza. En efecto, pareciera entonces que el concepto de transformative use integra no solo la modificación sustancial del contenido sino además el cambio de formato cuando este cumpla con las principales finalidades del derecho de autor y de la doctrina del fair use. Así pues, del análisis de la decisión podría deducirse que no es posible concluir infracción ante una digitalización de obras (cambio de formato) cuyo propósito sea diferente del que dio origen a la obra en físico (su venta al público) y permita además cumplir con los fines del derecho de autor, es decir la promoción de la ciencia y las artes útiles, tal como viene consagrado desde la Constitución americana.

El estado actual de las cosas invita a la academia a estar presta a analizar posteriores decisiones en este sentido, ya que se mantienen varios interrogantes por resolver. Por ejemplo, es pertinente preguntarse si ante cualquier cambio de

25. Iona Silverman, "The us Court of Appeals considers transformative use in Cariou v. Prince: how far is too far?”, Journal of Intellectual Property Law Practice, 2013, 8, pp. 755-757.

26. Mark A. Fischer, Duane Morris llp, "All's fair in copying the world's books", disponible en: http://www.mediabizbloggers.com/mark-fischer/Alls-Fair-in-Copying-theWorlds-Books----Mark-Fischer.html 
formato, y con mayor razón en los nuevos contextos digitales, ello podría considerarse como un uso transformativo y, por ende, justo.

Parecería que la actual situación jurisprudencial colocara a los futuros demandantes o autores en una situación de presunción legal del uso justo que solo sería desvirtuada ante una prueba contundente de un "uso no transformativo". Estos, entre otros, podrían ser algunos de los interrogantes que podrían heredarse de la decisión de Google.

Aunque los interrogantes se encuentran formulados de tal forma que podrían ser calificados como de "radicales", también es importante reconocer que los escenarios podrían ser diferentes. Así, es posible que en posteriores decisiones se aclare con más detalle que la parte demandada sigue conservando la carga de la prueba de un uso justo, es decir, de su defensa, y es factible que existan, además, escenarios donde sean evidentes los grandes beneficios a la sociedad derivados de dichas interpretaciones del "uso transformativo", permitiendo el progreso de la ciencia, la cultura y las artes.

En el transcurso del proceso, Ned Rosenthal, el abogado de Authors Guild, propuso un método alterno de valoración del transformative use, que aunque se aleja de la perspectiva acogida por las Cortes, merece una reflexión. Adujo el abogado que la evaluación del caso no debería estar basada en los beneficios que los usuarios obtienen de Google Books, sino en las ventajas competitivas y ganancias económicas obtenidas por Google como consecuencia del uso de las obras sin previa autorización de los autores y sin el debido reconocimiento pecuniario ${ }^{27}$. Esta resulta ser una posición que restringe, en cierta medida, la interpretación del transformative use y que por tanto debería ser evaluada y ponderada con los demás elementos de la doctrina con el fin de arribar a una equilibrada aplicación del fair use.

Junto a lo anterior, debe también advertirse la flagrante contradicción entre las consideraciones de la Corte en el caso Google Books y el razonamiento aducido por la Suprema Corte en el caso Tasini vs. The New York Times ${ }^{28}$. En efecto, en este caso se concluyó que la autorización expresa del autor era requerida siempre que se pretendiera cambiar de formato la obra original con el fin de incluirla en una base de datos ${ }^{29}$. La Corte determinó:

27. Ahmad Asir, Google Books Litigation Update, 2013. Ned Rosenthal manifestó: "evaluate the case not based on how users use Google Books, but by how Google uses the corpus of scanned works - as a commercial advantage for its search engine competitors, without having paid copyright owners". Disponible en: http://btlj.org/2013/11/10/google-books-litigation-update/

28. Tasini v. The New York Times, disponible en: http://www.law.cornell.edu/supct/ pdf/00-201P.ZO

29. Rafael Ríos Wilson, "Análisis del acuerdo inicial y sus enmiendas planteadas por Google a los autores y titulares de derechos, y su situación actual”, Propiedad Inmaterial, 2010, 14, pp. 277-301. 
We conclude that the Electronic Publishers infringed the Authors' copyrights by reproducing and distributing the Articles in a manner not authorized by the Authors and not privileged by \$201(c). We further conclude that the Print Publishers infringed the Author'copyrights by authorizing the Electronic Publishers to place the Articles in the Databases and by aiding the Electronic Publishers in that endeavor.

Del análisis que la Corte hace del transformative use se concluye que se trata de una posición que no solo amplía considerablemente los alcances de la doctrina del fair use, sino que además se constituye en una evidente contradicción de la jurisprudencia de la Suprema Corte en materia del cambio de formato de las obras. Además, tal como se señaló atrás, la decisión marcó un escenario de incertidumbre frente a la resolución de casos referidos a la digitalización en masa, por cuanto aún son inciertos los factores aplicables en todos los casos con el fin de determinar si un uso de la obra ha sido transformativo. Así, entonces, le corresponde a la academia auscultar las posibles consecuencias del caso Google Books en contextos en los cuales los nuevos retos tecnológicos han impuesto la necesidad de preguntarse si un cambio de formato constituye un uso transformativo. Por ello, en el siguiente acápite se estudian brevemente los posibles efectos de los fallos que se comentan en el sistema bibliotecario.

\section{IMPLICACIONES DE LA DECISIÓN EN UN UNIVERSO DE DIGITALIZACIÓN EN MASA, Y EN ESPECIAL, RESPECTO DE LOS PRÉSTAMOS BIBLIOTECARIOS}

El estudio de las decisiones en el caso Google Books plantea también un escenario de incertidumbre respecto del régimen de limitaciones y excepciones aplicable a las bibliotecas. Lo anterior se debe a la generalizada tendencia de crear repositorios digitales de obras, para de esta forma ofrecer al usuario nuevas modalidades de préstamos o de consultas. Así entonces, se han generado nuevos modelos de negocio y contratación en torno de las funciones desarrolladas por las bibliotecas.

El análisis de este contexto exige reconocer la importante función que las bibliotecas desempeñan en nuestra sociedad. En efecto, no solo consisten en grandes repositorios de conocimiento disponibles para ser consultados por parte de usuarios, investigadores, profesores y estudiantes, sino que además cumplen con fines de democratización del saber. Respecto de este último punto, debe traerse a colación la labor desempeñada por las bibliotecas públicas, las cuales, sin una remuneración previa proveniente del usuario, permiten el acceso indiscriminado a sus repositorios bibliográficos. Si bien las limitaciones y excepciones a favor de las bibliotecas, consagradas en la Sección 108 del Copyright Act americano, 
plasman la gran importancia que representan en nuestra sociedad, dicha norma no aclaró el régimen jurídico aplicable al préstamo de los e-book ${ }^{30}$.

En Estados Unidos, el préstamo bibliotecario se encuentra regido por el principio del agotamiento del derecho denominado the first sale doctrine. De acuerdo con esta doctrina, una vez el titular de los derechos ha autorizado la primera venta de su obra, posteriormente no se encuentra habilitado jurídicamente para objetar la disposición y redistribución de sus copias. Cabe resaltar que la mencionada doctrina nace a raíz del caso Bobbs-Merrill Co. v. Strauss, decisión en la cual la Suprema Corte, con base en la distinción entre la propiedad material y la inmaterial, concluyó que el derecho exclusivo que el ordenamiento jurídico les reconoce a los autores no implica que estos puedan prohibir posteriores ventas de la obra. La Corte se pronunció en los siguientes términos:

The copyright is distinct from the property in the material object copyrighted, and the sale or conveyance, by gift or otherwise, of the material object shall not of itself constitute a transfer of the copyright, nor shall the assignment of the copyright constitute a transfer of the title to the material object; but nothing in this title shall be deemed to forbid, prevent, or restrict the transfer of any copy of a copyrighted work the possession of which has been lawfully obtained ${ }^{\beta 1}$.

Lo anterior fue consagrado en la Sección 109 del Copyright Act de 1976, disposición que aunque no fijó literalmente la distinción entre propiedad inmaterial y material, previó la esencia de la first sale doctrine: "the owner of a particular copy or phonorecord [...] is entitled, without the authority of the copyright owner, to sell or otherwise dispose of the possession of that copy or phonorecord".

El agotamiento del derecho a través de esta doctrina permite que las bibliotecas desarrollen su actividad sin necesidad de obtener previa autorización de los titulares cada vez que se efectúe un préstamo o se realicen consultas por parte de los usuarios. Ahora bien, a nivel doctrinario y en ciertas regiones del planeta, el derecho de autor ha variado su dimensión, pasando de ser únicamente el derecho exclusivo de vender y distribuir la obra, a una concepción más amplia, en la cual se incluyó el derecho de realizar préstamos. Comoquiera que el derecho de préstamo empezó a concebirse en ciertas latitudes como parte de aquel conjunto de prerrogativas del autor, resultaría entonces necesario contemplar la remuneración previa del autor.

Así pues, ciertos autores han sostenido que el nuevo entorno digital exige de todos los sistemas jurídicos una consagración normativa específica del derecho

30. Matthew Chiarizio, "American tragedy: E-books, licenses, and the end of public lending libraries?”, 66 Vand. L. Rev. 615, 2013.

31. Ibíd. 
de alquiler o préstamo, denominado por la doctrina como el public lending, que contemple una remuneración previa del autor cada vez que la obra sea objeto de un préstamo, o al menos un sistema que se adapte correctamente a los nuevos retos tecnológicos ${ }^{32}$.

No obstante, el sistema del préstamo o "lending" basado en el principio del agotamiento del derecho a partir de la primera venta no es viable respecto de los e-book ya que su préstamo implica la reproducción de la obra. En efecto, dada su naturaleza electrónica, el préstamo requiere una reproducción constante. De la obra original se derivarán entonces varias copias digitales, una residirá en el sistema de la biblioteca y las demás estarán en el sistema de cada uno de los usua$\operatorname{rios}^{33}$. Adicionalmente, el agotamiento del derecho por la primera venta requiere precisamente que la obra haya sido adquirida por un comprador. Lo anterior no sucede respecto de los e-book debido a que su negociación se encuentra basada en el licenciamiento o autorización de uso, y no en la venta.

La imposibilidad de aplicar la doctrina de la primera venta ha generado incertidumbre acerca del régimen jurídico aplicable a los repositorios digitales de las bibliotecas. Por un lado, a diferencia de lo que sucede con el formato físico, las bibliotecas no son propietarias de las obras digitales y los derechos de disposición que cuentan sobre las mismas dependen directamente de los acuerdos de licencia celebrados con los autores o el término de suscripción acordado con los titulares de derechos. Aunado a lo anterior, dado que el nuevo entorno digital ha ocasionado que la mayoría de las obras se distribuyan en formato digital, esto podría derivar en problemas para las bibliotecas públicas por cuanto al no haberse agotado el derecho, los autores podrían objetar el libre acceso a las obras digitales y su reproducción ilimitada sin remuneración previa ${ }^{34}$.

Pese a las innumerables iniciativas legislativas -e incluso decisiones judicialestendientes a llenar este vacío jurídico, no se ha llegado a una verdadera solución. Por la falta de claridad en la regulación, los préstamos digitales son cada vez más frecuentes. En efecto, en el año 2011, el 82\% de las bibliotecas públicas en Estados Unidos optaron por ofrecer préstamos digitales a sus usuarios. Para ello, las bibliotecas se han venido asociando con la compañía Over Drive, la cual cumple funciones de intermediación entre los editores y las bibliotecas. En esencia, el modelo ofrece una dinámica en la cual los e-book son asimilados a las versiones físicas por cuanto se acuerdan cuantas licencias sean necesarias con el fin ofrecer un número determinado de copias a los usuarios. Así mismo, una vez se ha concedido el préstamo de la obra, no se permite a los demás usuarios consultar el mismo texto. Además, debido a los mecanismos tecnológicos de protección 
aplicados bajo este modelo, los usuarios no están habilitados para reproducir las obras o conservarlas después de expirado el término del préstamo ${ }^{35}$.

Una vez descrito el escenario de los préstamos digitales en Estados Unidos, es necesario indagar si realmente un nuevo régimen jurídico es requerido para garantizar mayor claridad y certeza en las relaciones jurídico negociales entre autores, bibliotecas y usuarios. Un sector de la doctrina responde negativamente ante tal inquietud y justifica su posición argumentando que una intervención del Estado en dichas relaciones y en la dinámica impuesta por los nuevos modelos de negocios del e-book sería perjudicial para la difusión del conocimiento y la promoción de la creatividad. Matthew Chiarizio explica que restringir la creatividad en la construcción de nuevos modelos como el propuesto a través de la compañía Over Drive ocasionaría un grave retroceso en el desarrollo de los fines últimos del derecho de autor: promover la creación mientras se garantiza el acceso al conocimiento ${ }^{36}$.

En cuanto al caso Google Books, aunque las Cortes no lo mencionan expresamente, pareciera que sus considerandos estuvieran también orientados a estimar que todo cambio de formato realizado por las bibliotecas, con el fin de promover el acceso al conocimiento a través de sus sistemas o el de terceros como Google, constituiría un transformative use. Ahora bien, podría considerarse que esta interpretación no soluciona la incertidumbre existente en torno del régimen jurídico aplicable a los préstamos digitales, ampliándose además el espectro de los usos justos por parte de las bibliotecas y sus usuarios. Lo anterior se configura entonces en una invitación a la jurisprudencia y a la academia a esclarecer el régimen jurídico aplicable, el cual debe balancear todos los intereses involucrados.

Si bien el transformative use ha resultado ser una doctrina que flexibiliza al extremo los derechos de autor, dejando interrogantes señalados en los acápites anteriores, no es posible desconocer la elemental función de democratización del conocimiento desempeñada tanto por Google como por las bibliotecas. En consecuencia, es necesario también darle protagonismo a las grandes ventajas derivadas de proyectos como el de Google Books. Y es que dicho sistema ha permitido que poblaciones menos desarrolladas y en desarrollo conozcan la existencia de obras a las cuales no habrían podido acceder de otra forma, salvo que se hubieran adquirido bajo alguna modalidad de suscripción con las bibliotecas partícipes del proyecto $^{37}$. Sobre este punto, vale la pena mencionar una importante frase de Nelson Mandela, en la cual logró plasmar la importancia de garantizar el acceso

35. Ibíd.

36. Ibíd.

37. Douglas L. Rogers, Vorys, Sater, Seymour and Pease llp, "Access to knowledge as a bridge over the troubled waters of Copyright Fair Use -- From Jefferson to Mandela to Google", Bepress Legal Series, disponible en: http://law.bepress.com/expresso/eps/1649/ 
al conocimiento por parte de todas las poblaciones, como factor necesario para mejorar la calidad de vida de la humanidad: "eliminating the distinction between the information-rich and information-poor is also critical to eliminating economic and other inequalities between North and South, and to improving the life of all bumanity" 38 .

Dadas las ventajas derivadas del entorno digital como mecanismo difusor del conocimiento, no es posible adoptar posiciones radicales respecto de la extensión de los derechos. Así pues, aunque debe reconocerse la importancia de las excepciones y limitaciones al derecho de autor, estas deben encuadrase en concepciones mucho más claras y certeras que permitan al juez concluir, en un entorno de seguridad jurídica, si determinada conducta debe considerarse como infractora o no. Lo anterior ofrecería además un contexto mucho más claro para la contratación y disposición de obras digitales por parte de las bibliotecas.

\section{Conclusiones}

Las sentencias de Google plasman en sus considerandos la evolución del derecho de autor, concebido e interpretado en el marco de una nueva era marcada por fuertes cambios tecnológicos. Si bien estas decisiones no ponen punto final a la discusión y, por el contrario, abren compuertas al debate y a posteriores interpretaciones, también es cierto que se suman a un conjunto de decisiones jurisprudenciales que admiten una extensiva digitalización de obras y que lo enmarcan en un uso justo, benéfico para la sociedad y sin que esto configure violaciones a los derechos de autor. Esto podría promover mayor innovación en la difusión de la información en internet a través de mecanismos que beneficien a la sociedad sin cercenar derechos de autor de manera irremediable.

Ahora bien, del análisis de las sentencias se encuentra también que la aplicación de la doctrina del fair use y ahora del concepto del transformative use puede generar cierta ambigüedad en la protección de los derechos de autor. En efecto, se trata de concepciones jurisprudenciales que dependen exclusivamente de fundamentos fácticos, es decir que, así como en este caso se concluyó que la conducta se enmarcaba dentro de dichos conceptos, puede que en un caso similar se llegue a diferentes decisiones determinadas por las circunstancias en concreto. Esto, aunque permite al derecho de autor adecuarse a las nuevas realidades impuestas por la era digital, también implica incertidumbre sobre la interpretación adecuada en casos como el que se comenta.

Resulta relevante anotar que Google no solo recibe autorización para continuar digitalizando extractos de obras, sino que además se le otorga una gran ventaja competitiva que la ubica una vez más entre los líderes o quizá la empresa 
más poderosa en tecnologías de la información. Aunque esta decisión podría abrir grandes posibilidades para otros buscadores, como Yahoo, para emprender proyectos como el de Google Books, este último cuenta ya con una base de datos de dimensiones insuperables. Google tiene el control exclusivo sobre un gran índice de libros que viene construyendo desde el año 2004 y no resultaría atractivo para sus competidores tratar de equiparar dicho sistema ya que no solo implicaría grandes inversiones de dinero, sino que además abriría la posibilidad a posteriores conflictos jurídicos y cuantiosas demandas.

Además, surgen otros interrogantes que influyen directamente en el devenir del nuevo derecho de autor. Por un lado, debe consultarse también los efectos que las sentencias tienen respecto de la regulación y análisis de las obras huérfanas. Efectivamente, en las sentencias se omite el análisis específico de la explotación de dichas obras comoquiera que a juicio de la Corte del Distrito se trata de un asunto que no debe tratarse con efectos inter partes sino erga omnes y, por ende, el Congreso es el ente competente para regular la materia. No obstante, generan dudas los resultados prácticos de lo anterior dado que aunque no se resuelve el vacío jurídico existente con relación a esta categoría de obras, sí se admite su digitalización y difusión en internet sin ningún tipo de restricción ${ }^{39}$.

Adicionalmente, aunque la Corte de Apelaciones concluye que no existe responsabilidad contributiva ${ }^{40}$, por cuanto se trata de un supuesto hipotético según el cual las bibliotecas puedan infringir los derechos de autor, es aún un misterio el régimen de responsabilidad aplicable en el caso de Google Books y en el supuesto real en el cual las bibliotecas efectivamente infrinjan los derechos. En efecto, debe determinarse en el futuro si Google es responsable por las descargas de contenidos que las bibliotecas realizan a raíz del sistema creado. Ante una respuesta afirmativa, convendría también consultar si se trata entonces de una responsabilidad directa (direct copyright infringement), contributiva (contributory copyright infringement) o vicaria (vicarious copyright infringement $)^{41}$. Las mismas inquietudes surgirían respecto de la responsabilidad de las bibliotecas al haber celebrado acuerdos con Google sin previa autorización de los autores con la finalidad de crear el gran índex de libros que posteriormente reconoceríamos como "Google Books".

39. Eleonora Rosati, "Google Books' Library Project is fair use", University of Cambridge, Journal of Intellectual Property Law and Practice, 2014, vol. 9, n. ${ }^{\circ}$ 2, p. 102.

40. "There is no basis in the record to impose liability on Google for having lawfully made a digital copy for a participating library so as to enable that library to make non-infringing use of its copy, merely because of the speculative possibility that the library may fail to guard sufficiently against the dangers of hacking, as it is contractually obligated to do [...] Nor, on this record, is Google a contributory infringer".

41. Para mayor información sobre estos conceptos cfr. "El derecho de autor y los derechos conexos en el entorno digital", disponible en: http://webcache.googleusercontent. $\mathrm{com} / \mathrm{search}$ ?q=cache:utPD4YCaEEgJ:www.wipo.int/edocs/mdocs/lac/es/ompi_ 
En un contexto de globalización como el actual, los efectos de una determinada decisión repercuten inmediatamente en los desarrollos jurídicos de otras latitudes, y esto sucede con mayor razón respecto de Estados Unidos, la economía líder mundial. En consecuencia, le corresponde a la academia también evaluar qué alcances tendrá la interpretación del transformative use en otros ordenamientos jurídicos como el colombiano. Esto no solo interesa debido a la influencia del derecho americano en la protección de la propiedad intelectual, sino además debido a los distintos niveles de desarrollo involucrados. La extensión o limitación de los derechos en un país en desarrollo podría tener mayores efectos colaterales en la economía, educación y acceso al conocimiento que los generados en las regiones más desarrolladas.

En el ordenamiento jurídico colombiano no existe una doctrina como la del fair use y por ende, no resultan aún completamente claros los efectos que tendría la decisión de Google Books. Ahora bien, cabe anotar que, en virtud del artículo 22 literal c) de la Decisión Andina 351 de 1993, y el artículo 38 de la Ley 23 de 1998, es posible realizar la reproducción de una obra sin previa autorización y remuneración del autor, cuando sea necesario para el desarrollo de las funciones de las bibliotecas. Es decir, dicha excepción tendrá cabida cuando se pretenda preservar algún ejemplar o sustituirlo en caso de extravío, destrucción o inutilización, o para préstamos a otras bibliotecas.

Tal como lo ha señalado la Dirección Nacional de Derechos de Autor colombiana, las anteriores disposiciones deben interpretarse bajo el entendido de que la digitalización de una obra debe considerarse como una reproducción. Así mismo, ha señalado la entidad que el cambio de formato, entendido como reproducción en el ámbito de aplicación de las normas señaladas, no debe afectar la normal explotación de la obra ni causar un perjuicio injustificado a los derechos del titular ${ }^{42}$. Aunque estos últimos factores podrían subsumirse en el análisis que se hace del fair use en Estados Unidos, es necesario señalar que en nuestro ordenamiento las circunstancias en las cuales es admitido el cambio de formato son más claras y expresamente determinadas por la norma. Así entonces, el proyecto de Google Books no pareciera estar acorde con nuestro ordenamiento. Si bien podría configurarse dentro de las limitaciones y excepciones a favor de las bibliotecas, las normas correspondientes encuadran el cambio de formato dentro de un campo de acción limitado, para ciertos fines determinados, y para la consulta de los usuarios de las bibliotecas, pero no con el fin de permitir la consulta por parte de todo aquel que consulte el amplio espectro universo de internet.

Por último, y para mayor claridad, cabe destacar la posición del profesor Fernando Zapata López, quien en unas pocas líneas explica las circunstancias en 
las cuales el cambio de formato puede considerarse acorde con el ordenamiento: "las bibliotecas están en capacidad de reproducir y difundir públicamente obras literarias y artísticas en cuatro casos: cuando la biblioteca sea titular de derechos de las obras, cuando dichas obras se encuentren en el dominio público (revisar la legislación de cada país), cuando la digitalización se encuentre amparada por una limitación o excepción de derecho de autor, o cuando se haya obtenido la licencia de uso por parte del autor o titular de la obra”“3.

Pese a los grandes impactos de la decisión de Google, los expertos se encuentran positivos a la espera del eco que esta decisión pueda tener en posteriores decisiones jurisprudenciales. Algunos sostienen que posiciones como la asumida en este caso promoverán mayor innovación y creación. Lo cierto es que la doctrina del transformative use podría ser una gran puerta para cualquier cambio de la obra original, situación que genera mayor ambigüedad en el entorno digital dado que cualquier cambio de formato con beneficios sociales puede ser considerado un uso justo de la obra.

\section{Bibliografía}

Adelstein Richard y Peretz Steven, "The competition of technologies in markets for ideas: Copyright and fair use in evolutionary perspective", International Review of Law and Economics, 1985, disponible en: http://works. bepress.com/cgi/viewcontent. cgi?article $=1015 \&$ context=adelstein\&sei-redir $=1$

Authors Guild v. Google, Inc. United States Court of Appeals for The Second Circuit - October 16, 2015.

Denny Chin, United States District Court Southern District of New York, The Authors Guild et al. v. Google Inc, decisión del 22 de marzo de 2011, disponible en: http://www.nysd.uscourts.gov/cases/show.php?db=special\&id=115

Dirección Nacional de Derechos de Autor, Concepto Jurídico Rad. 1-2006-18058, Disponible en: P:IJuridicalConceptos\2010\2-2010-47984

Griffiths, Jonathan, "Unsticking the centre-piece - The liberation of European copyright law", disponible en: http://www.jipitec.eu/issues/ jipitec-1-2-2010/2617/JIPITEC\%202\%20-

Halpern, Sheldon, Seymore Sean y Port Kenneth, Fundamentals of United States Intellectual Property Law, 4. ${ }^{\mathrm{a}}$ ed., Wolters Kluwer, uK, 2012.

Herrera Sierra, Luisa Fernanda, "Estudio del Caso Google Books y efectos del fair use en el entorno digital", disponible en: http://propintel.uexternado.edu. co/estudio-del-caso-google-books-y-efectos-del-fair-use-en-el-entorno-digital/ 
Kimbrough, Ashten, "Transformative use vs. market impact: Why the fourth fair use factor should not be supplanted by transformative use as the most important element in a fair use analysis", Alabama Law Review, 2012.

Mark A. Fischer, Duane Morris llp, "All's fair in copying the world's books", disponible en: http://www.mediabizbloggers.com/mark-fischer/Alls-Fair-inCopying-the-Worlds-Books----Mark-Fischer.html

Murray, Michael D., "What is transformative? An explanatory synthesis of the convergence of transformation and predominant purpose in Copyright Fair Use Law", Valparaiso University School of Law, 11 Chi.-Kent J. Intell. Prop. 260 (2012).

OMPI, "El derecho de autor y los derechos conexos en el entorno digital", disponible en: http://webcache.googleusercontent.com/search?q=cache:utPD4YCaEEgJ:www. wipo.int/edocs/mdocs/lac/es/ompi_

Rosati, Eleonora, "Google Books' Library Project is fair use", University of Cambridge, Journal of Intellectual Property Law and Practice, 2014, vol. 9, n. 2. Seth, Godin, "The big mistake we all make about ideas", disponible en: http:// blog.ted.com/2014/02/03/the-big-mistake-we-all-make-about-ideas/

Silverman, Iona, "The us Court of Appeals considers transformative use in Cariou v. Prince: How far is too far?”, Journal of Intellectual Property Law Practice, 2013, 8, pp. 755-757.

Zapata López, Fernando, "La digitalización de las bibliotecas públicas debe ir de la mano del derecho de autor", disponible en: http://cerlalc.org/unescola-digitalizacion-de-las-bibliotecas-publicas-debe-ir-de-la-mano-del-derechode-autor/ 